\title{
Involvement of A5/A7 noradrenergic neurons and B2 serotonergic neurons in nociceptive processing: a fiber photometry study
}

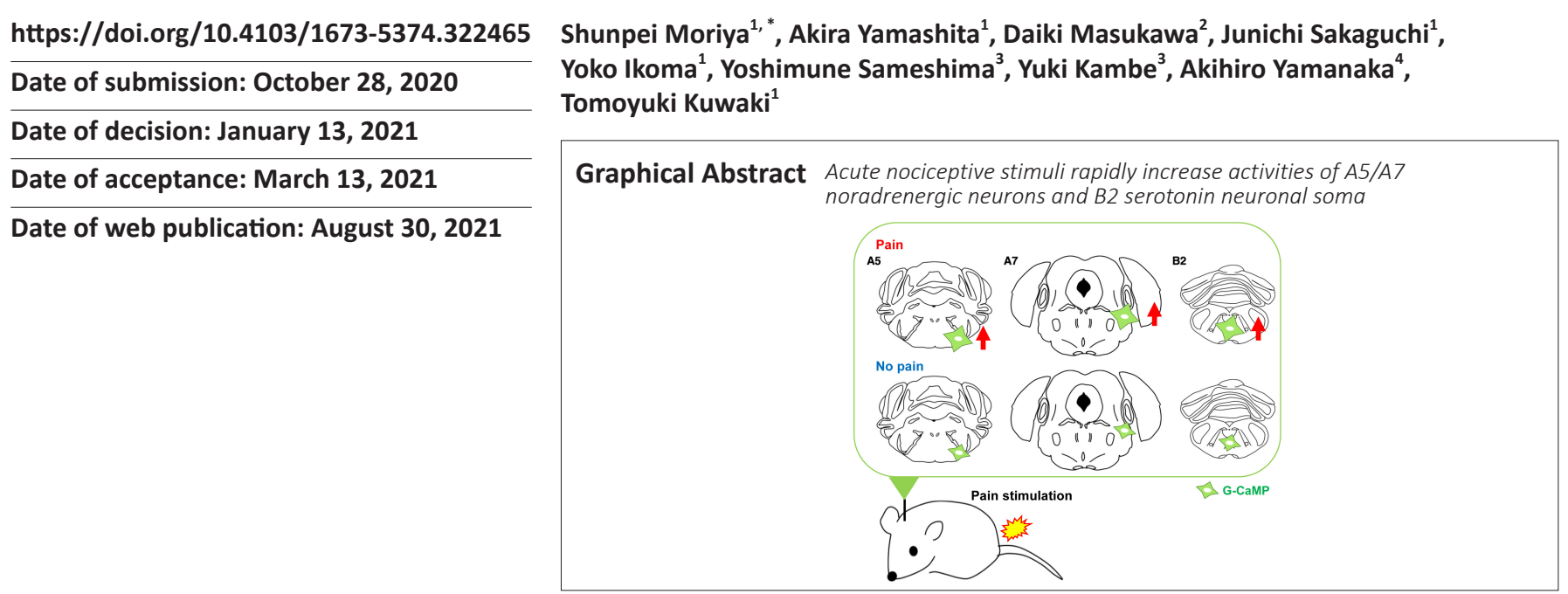

\begin{abstract}
In the central nervous system, the A6 noradrenaline (NA) and the B3 serotonin (5-HT) cell groups are well-recognized players in the descending antinociceptive system, while other NA/5-HT cell groups are not well characterized. A5/A7 NA and B2 5-HT cells project to the spinal horn and form descending pathways. We recorded G-CaMP6 green fluorescence signal intensities in the A5/A7 NA and the B2 5-HT cell groups of awake mice in response to acute tail pinch stimuli, acute heat stimuli, and in the context of a non-noxious control test, using fiber photometry with a calcium imaging system. We first introduced G-CaMP6 in the A5/A7 NA or B2 5-HT neuronal soma, using transgenic mice carrying the tetracycline-controlled transactivator transgene under the control of either a dopamine $\beta$-hydroxylase or a tryptophan hydroxylase-2 promoters and by the site-specific injection of adeno-associated virus (AAV-TetO(3G)-G-CaMP6). After confirming the specific expression patterns of G-CaMP6, we recorded G-CaMP6 green fluorescence signals in these sites in awake mice in response to acute nociceptive stimuli. G-CaMP6 fluorescence intensity in the A5, A7, and B2 cell groups was rapidly increased in response to acute nociceptive stimuli and soon after, it returned to baseline fluorescence intensity. This was not observed in the non-noxious control test. The results indicate that acute nociceptive stimuli rapidly increase the activities of A5/A7 NA or B2 5-HT neurons but the non-noxious stimuli do not. The present study suggests that A5/A7 NA or B2 5-HT neurons play important roles in nociceptive processing in the central nervous system. We suggest that A5/A7/B2 neurons may be new therapeutic targets. All performed procedures were approved by the Institutional Animal Use Committee of Kagoshima University (MD17105) on February 22, 2018.
\end{abstract}

Key Words: A5 NA neurons; A7 NA neurons; B2 5-HT neurons; DBH-tTA mice; fiber photometry; G-CaMP6; mCherry; monoaminergic signaling; nociception; TPH-tTA mice

Chinese Library Classification No. R448; R364; R741

\section{Introduction}

Pain symptoms frequently present in various mental and physical disorders and are sometimes difficult to treat (Makris et al., 2014). The role of monoaminergic signaling in nociceptive processing in the central nervous system (CNS) is well known. Part of the pain management system is the descending antinociceptive system (DAS) (Melzack and Wall 1965; Costa et al., 2019), whose main pathways are the noradrenergic (NAergic) locus coeruleus (A6)-spinal horn circuit and serotonergic (5-HTergic) periaqueductal greyrostral ventromedial medulla (B3)-spinal horn circuit (Yakch et al., 1981; Hirschberg et al, 2017). In the CNS, the subsets of noradrenaline (NA) cells are classified into groups A1-A7 from the caudal to the rostral direction (Dahlstrom and Fuxe, 1964; Pertovaara, 2006). The A6 NA cell group is a well-recognized player in the DAS (Yaksh et al., 1981), while other NA cell groups are not well characterized. However, a few studies have reported that A5 and A7 NA cell groups were implicated

${ }^{1}$ Department of Physiology, Kagoshima University Graduate School of Medical and Dental Science, Kagoshima, Japan; ${ }^{2}$ Department of Molecular Pharmacology and Neurobiology, Yokohama City University Graduate School of Medicine, Yokohama, Japan; ${ }^{3}$ Department of Pharmacology, Kagoshima University Graduate School of Medical and Dental Science, Kagoshima, Japan; ${ }^{4}$ Research Institute of Environmental Medicine, Nagoya University, Nagoya, Japan

${ }^{*}$ Correspondence to: Shunpei Moriya, MD, PhD, msmr9040psyg80@gmail.com.

https://orcid.org/0000-0002-2242-8214 (Shunpei Moriya)

Funding: This work was supported by JSPS KAKENHI grants (Nos. 19 K17093 to SM; 20 K06858 to AYamashita; 16H05130 to TK) and CREST JST (No. JPMJCR1656 to AYamanaka).

How to cite this article: Moriya S, Yamashita A, Masukawa D, Sakaguchi J, Ikoma Y, Sameshima Y, Kambe Y, Yamanaka A, Kuwaki T (2022) Involvement of A5/A7 noradrenergic neurons and B2 serotonergic neurons in nociceptive processing: a fiber photometry study. Neural Regen Res 17(4):881-886. 


\section{Research Article}

in the regulation of antinociception (Nuseir and Proudfit, 2000; Mravec et al., 2012). The A5 cell group is located in the ventrolateral part of the pons and the A7 cell group is located in the lateral part of the pons (Proudfit and Clark, 1991). The A5/A7 and A6 NA cells mainly project into the superficial spinal horn (Proudfit and Clark, 1991). The 5-HTergic and NAergic systems have extensive innervations in the CNS; the subsets of serotonin (5-HT, 5-hydroxytryptamine) cells are classified as groups B1-B9 from the caudal to the rostral direction (Dahlstrom and Fuxe, 1964). Although the B3 5-HT group is a known player in the DAS, the other $5-\mathrm{HT}$ cell groups have not been studied sufficiently well (Jacobs and Azmitia, 1992). The B2 (nucleus raphe obscurus) 5 -HT cell group is located in the ventral part of the medullary reticulum (Jacobs et al., 2002). Unlike the B3 group, the B2 group mainly projects into the spinal intermediolateral cell column (IML) (Allen and Cechetto, 1994; Benarroch, 2014) and spinal ventral horn (Saruhashi et al., 1996). The B2 group regulates the autonomic (Allen and Cechetto, 1994; Benarroch, 2014) and motor function (Saruhashi et al., 1996; Benarroch, 2014). Few studies have assessed the contribution of the $\mathrm{B} 2$ group in regulating nociceptive processing. Recently, it has been reported that the activities of A6 NA neurons and B3 5-HT neurons in awake mice are rapidly increased in response to acute nociceptive stimuli measured using a fiber photometry system (Moriya et al., 2019). In this study, we have chosen the A5/A7 and B2 cell groups to explore the role of monoaminergic signaling in DAS. Fiber photometry with a calcium $\left(\mathrm{Ca}^{2+}\right)$ imaging system is an advanced tool for evaluating nociceptive signaling; this was demonstrated in a recent study focused on B9 5-HT (Moriya et al., 2020a) and A13 dopamine (DA) neurons (Moriya et al., 2020b). Therefore, we assessed the activities of A5/A7 NA and B2 5-HT neurons in response to acute nociceptive stimuli.

\section{Materials and Methods}

\section{Animals}

We used transgenic mice carrying tetracycline-controlled transactivator transgene (tTA) under the control of a dopamine $\beta$-hydroxylase $(\mathrm{DBH})(24.0 \pm 1.0 \mathrm{~g}, n=12)$ or a tryptophan hydroxylase-2 (TPH2) promoters $(23.8 \pm 1.1 \mathrm{~g}, n=6)$ (DBH-tTA mice or TPH2-tTA mice, respectively, from Research Institute of Environmental Medicine, Nagoya University, Nagoya, Japan) (Moriya et al., 2018, 2019, 2020a) (Figure 1A); 10-14-weekold male mice were used in this experiment). Rearing was carried out in standard conditions, with lights on at 7:00 a.m. and off at 7:00 p.m., a temperature of $24 \pm 1^{\circ} \mathrm{C}$, and food and water available ad libitum. We made efforts to minimize animal suffering and reduce the number of animals used. All experimental procedures were performed following the National Institute of Health Guide for the Care and Use of Laboratory Animals and approved by the Institutional Animal Use Committee of Kagoshima University (MD17105) on February 22, 2018.

\section{Stereotaxic adeno-associated virus injection}

Adeno-associated virus ( $A A V$ ) vectors were produced using the AAV Helper-Free system (Agilent Technologies, Inc., Santa Clara, CA, USA) and purified as described previously (Inutsuka et al., 2016). AAV-TetO(3G)-G-CaMP6 (Serotype: DJ; $0.3 \mu \mathrm{L} /$ injection, $4 \times 10^{13}$ copies $/ \mathrm{mL}$ ) and AAV-Tet(3G)-mCherry (Serotype: DJ; $0.3 \mu \mathrm{L} /$ injection, $6 \times 10^{12}$ copies $/ \mathrm{mL}$ ) were produced using the Tet system (Figure 1B). The specific sequences of AAV vectors are provided (Additional file $\mathbf{1}$ ). We slowly siphoned AAV into a glass micropipette (1B150F-3, World Precision Instruments, Inc., Sarasota, FL, USA) connected to an injection manipulator (I-200J, Narishige, Tokyo, Japan) linked to a nitrogen pressure source through a polyethylene tubing. Under the influence of $2-3 \%$ of isoflurane (Pfizer Inc., New York, NY, USA) as the inhalation anesthetic, the mice were fixed with a stereotaxic instrument (ST-7, Narishige) with the help of a supportive ear bar (EB-6, Narishige). To minimize suffering, the surfaces of their ears were covered with a local anesthetic jelly (lidocaine, 2\% xylocaine [Fujifilm Wako Pure Chemical Inc., Osaka, Japan]) and both eyes were preserved with Vaseline. Head hair was shaved using an electric shaver and the cranial dura mater was cut open with small scissors. We chose target coordinates according to Paxinos and Flanklin's the Mouse Brain in Stereotaxic Coordinates, Fifth Edition (Paxinos and Franklin, 2019). AAV was unilaterally injected into the target sites (A5: bregma $-5.52 \mathrm{~mm}$, lateral $+1.4 \mathrm{~mm}$, and ventral $-5.30 \mathrm{~mm}$ from the cranium; A7: bregma $-4.96 \mathrm{~mm}$, lateral $+1.88 \mathrm{~mm}$, and ventral $-3.10 \mathrm{~mm}$ from the surface of the brain; B2: bregma $-7.56 \mathrm{~mm}$ and ventral $-3.93 \mathrm{~mm}$ from the cranium) (Figure 1C) and the glass microtube was left in the sites for 10 minutes before being withdrawn. Postoperative antibiotic administration was carried out by subcutaneous injection (penicillin G: 40,000 U/kg) to prevent postoperative infections. After operation, the mice were maintained at standard rearing conditions (as described above) for 14 days to recover from the surgery and to allow the G-CaMP6/mCherry fluorescent protein to be fully expressed before the experimental sessions.

\section{Immunohistochemistry}

After the experiment, we performed immunohistochemistry to confirm the AAV-induced site-specific expression of G-CaMP6 and mCherry in mice. We perfused the mice transcardially with $20 \mathrm{~mL}$ of phosphate-buffered saline (PBS) and $20 \mathrm{~mL}$ of $4 \%$ paraformaldehyde solution under anesthesia with urethane $(1.6 \mathrm{~g} / \mathrm{kg}$, i.p.). The brain was removed, fixed with $4 \%$ paraformaldehyde, and soaked in $30 \% \mathrm{w} / \mathrm{v}$ of sucrose in PBS for 2 days. We created a series of $30 \mu \mathrm{m}$ slices, including the target sites with a cryostat (Cryotome FSE, Thermo Scientific, Yokohama, Japan), and immersed the sections in PBS for 24 hours at $4^{\circ} \mathrm{C}$. Every third section was used for analysis. We washed the sections with PBS three times and incubated them with primary antibodies overnight at $4^{\circ} \mathrm{C}$. We diluted the primary antibodies in blocking buffer at the following concentrations: A5/ A7: rabbit anti-tyrosine hydroxylase (TH) (AB152, EMD Millipore Corp., Temecula, CA, USA), 1:500; B2: sheep anti-TPH antibody (AB1541, EMD Millipore Corp.), 1:1000. The next day, we washed the sections with PBS three times and incubated them with secondary antibodies at room temperature for 2 hours. We diluted the secondary antibodies in PBS at the following concentrations: A5/A7: CF647 donkey anti-rabbit (20047, Biotium Inc., Fremont, CA, USA), 1:200; B2: CF647 donkey anti-sheep (20284, Biotium Inc.), 1:200. Next, we washed the sections with PBS once, mounted them on microscope slides (PRO-02, Matsunami, Osaka, Japan), and covered them with a micro cover glass (C024601, Matsunami). We observed the sections under a fluorescence microscope (BZ-X700, Keyence, Osaka, Japan) and created images using the Adobe Photoshop CC software (Adobe Systems Inc., San Jose, CA, USA).

\section{In vivo fiber photometry system}

In this study, we used the same fiber photometry system with two channels that was used in previous studies (Moriya et al., $2018,2019,2020 a, b)$. The scheme of the fiber photometry system is illustrated in Figure $\mathbf{2 A}$. In brief, a high-power LED driver (LEDD1B/M470F3, Thorlabs Inc., Newton, NJ, USA) generated an excitation blue light $(470 \mathrm{~nm}$ ) or yellow light (590 $\mathrm{nm}$ ) of power $0.1 \mathrm{~mW}$ at the tip of the silica fiber; the blue or yellow light passed the excitation bandpass filter (passbandblue light: $475 \pm 12.5 \mathrm{~nm}$; yellow light: $590 \pm 12.5 \mathrm{~nm}$ ), was reflected by a dichroic mirror-1, and joined the single silica fiber (diameter: $400 \mu \mathrm{m}$; numerical aperture: 0.6). The blue/ yellow light emitted from the tip of the silica fiber reflected the G-CaMP6/mCherry fluorescent proteins; the reflected green/red fluorescence signals were detected and transmitted to the same tube. The signals passed the dichroic mirror-1, were reflected by dichroic mirror-2, and passed the bandpass emission filter (passband-green: $510 \pm 12.5 \mathrm{~nm}$; red: $607 \pm$ $12.5 \mathrm{~nm}$ ). Finally, the signals were guided to a photomultiplier 
tube (PMTH-S1-1P28, Zolix Instruments, Beijing, China). The signals were digitized by an A/D converter (PowerLab8/35, AD Instruments Inc., Dunedin, New Zealand) and recorded with the LabChart version-7 software (AD Instruments Inc.).

\section{Experimental protocol}

In this study, we divided the experimental mice into three groups: $A 5, A 7$, and $B 2$ groups. Fourteen days prior to recording, $A A V$ was unilaterally injected into the $A 5 / A 7$ regions in DBH-tTA mice and B2 region in TPH2-tTA mice (Figure 1C). Each mouse was individually maintained in standard breeding conditions for 14 days after the operation. In this study, we recorded the G-CaMP6/mCherry green/red fluorescence intensities of A5/A7 NA and B2 5-HT neuronal cell bodies in response to acute nociceptive stimuli.

We carried out the recording according to the following procedure. Each mouse was anesthetized using 2-3\% isoflurane using a vaporizer and fixed with a stereotaxic instrument (ST-7, Narishige) using a supportive ear bar (EB6 , Narishige). Surfaces of the ears were smeared with a local anesthetic jelly (lidocaine, $2 \%$ xylocaine) to minimize suffering. We then carried out the silica fiber implantation operation. The silica fiber was placed just above the A5 site (bregma $-5.52 \mathrm{~mm}$, lateral $+1.4 \mathrm{~mm}$, and ventral $-5.30 \mathrm{~mm}$ from the cranium), A7 site (bregma $-4.96 \mathrm{~mm}$, lateral $+1.88 \mathrm{~mm}$, and ventral $-3.10 \mathrm{~mm}$ from the surface of the brain), and B2 site (bregma $-7.56 \mathrm{~mm}$ and ventral $-3.93 \mathrm{~mm}$ from the cranium). The coordinates were confirmed according to Paxinos and Flanklin's the Mouse Brain in Stereotaxic Coordinates, Fifth edition (Paxinos and Franklin, 2019). We implanted the silica fiber slowly while monitoring the fluorescence signal intensity; the fluorescence intensity was increased rapidly when the optical position approached the target sites (Figure 2B). After implantation, we stopped administering anesthesia. We waited for 2 hours after ceasing anesthesia for the first stimulus when the possible effects of anesthesia were reduced. To reduce any possible effects of a previous stimulus, we set the inter-stimulus interval to 30 minutes; the order of stimuli was as follows: first was the low heat stimulus $\left(25^{\circ} \mathrm{C}\right)$, second gentle touch, third the heat stimulus $\left(55^{\circ} \mathrm{C}\right)$, and last the pinch stimulus (Figure $\mathbf{2 C}$ ). We used the pinch meter (PM201, Soshin-Medic, Chiba, Japan) for the tail pinch stimulus and attached the apparatus to the root of the tail for three seconds with a force of $400 \times g$. We also used a heating probe (5R7-570, Oven Industries, Inc., Mechanicsburg, PA, USA), for the heat stimulus, set at $55^{\circ} \mathrm{C}$, and attached the probe to the root of the tail for three seconds. For non-noxious control stimuli, we used the same heat probe for the low heat stimulus and a cotton stick for the gentle touch; these stimuli were attached to the root of the tail for three seconds.

We defined the neuronal activity characteristic index as follows: F-the averaged fluorescence signal intensity value measured for three seconds just before each stimulus and defined as $100 \% ; \Delta \mathrm{F}$ : the maximum fluorescence signal intensity value during each stimulus, F; onset latency: the time from the start of the stimulus to the time when the fluorescence signal intensity exceeded the maximum value during the baseline period; peak latency: the time from the start of the stimulus to the maximum peak signal intensity.

\section{Statistical analysis}

Statistical analyses were conducted using a two-way analysis of variance (ANOVA) with Sidak's test for post hoc analyses. The two factors influencing $\Delta \mathrm{F} / \mathrm{F}$ were the mode (mechanical $v s$. thermal) and intensity (nociceptive vs. control) of the stimulus. The two factors influencing onset/peak latency were mode of the stimulus (pinch vs. heat) and target sites (A5 vs. A7 vs. B2). Data values were expressed as means \pm standard errors of the mean (SEM). Probability values of $P<0.05$ were considered statically significant. Analyses were performed using GraphPad Prism version 7 (GraphPad software, San Diego, CA, USA).

\section{Results}

Expression patterns of AAV-induced G-CaMP6/mCherry

The specific expression patterns of G-CaMP6/mCherry were confirmed in the A5/A7 NA (Figure $\mathbf{3 A}$ and $\mathbf{B}$ ) and B2 5-HT neuronal soma (Figure $\mathbf{3 C}$ ). G-CaMP6/mCherry-positive neurons were rarely observed outside the $A 5, A 7$, and $B 2$ areas. $\mathrm{TH}$-positive cells were found in the $\mathrm{A} 5$ and $\mathrm{A} 7$ areas. $A$ large proportion of them expressed G-CaMP6 (A5 area: 90.6\%, A7 area: 90.9\%; $n=6$, each; Figure 3D and E). All G-CaMP6 positive cells expressed mCherry, and a large proportion of them expressed TH (A5 area: 97.0\%, A7 area: 85.7\%; Figure 3D and $\mathbf{E}$ ). TPH-positive cells were found in the B2 area. A large proportion of them expressed G-CaMP6 (91.8\%; $n=6$; Figure 3F), and all G-CaMP6-positive cells expressed mCherry, while 94.3\% expressed TPH (Figure 3F). Therefore, green/red fluorescence was confirmed to be derived from specific A5/ A7/B2 sites.

\section{Effects of acute nociceptive stimuli on G-CaMP6/mCherry fluorescence intensity}

We histologically confirmed that the fiber was properly positioned (Additional Figure 1). G-CaMP6/mCherry fluorescence intensity was abruptly increased when the optical position was just above the target sites (Figure 2C). Figure 4 shows the averaged traces of G-CaMP6/mCherry fluorescence intensities in response to acute nociceptive stimuli or a nonnoxious control test ( $n=6$, each). ANOVA revealed that the G-CaMP6 fluorescence intensity was significantly different across different stimulus intensities (A5 group: pinch vs. touch: $F_{(1.5)}=36.84, P=0.0018$, heat vs. low heat: $F_{(1,5)}=24.28, P=$ $0.0044 ;$ A7 group: pinch vs. touch: $F_{(1,5)}=12.36, P=0.0170$, heat vs low heat: $F_{(1,5)}=11.48, P=0.0195$; $B 2$ group: pinch vs touch: $F_{(1,5)}=50.10, P=0.0009$, heat vs. low heat: $F_{(1,5)}=$ 23.81, $P=0.0046$; Figure 5A). ANOVA revealed no significant differences in onset/peak latency values across stimulus intensities (onset latency: pinch vs. touch: $F_{(2,10)}=0.3465, P$ $=0.7153$; heat vs low heat: $F_{(2,10)}=0.1266, P=0.8825$; peak latency: pinch vs. touch: $F_{(2,10)}=0.4141, P=0.6718$; heat vs. low heat: $\left.F_{(2,10)}=0.1148, \stackrel{P}{P}=0.8927\right)$. ANOVA revealed that the mCherry fluorescence intensity was not significantly different across stimulus intensities (A5 group: pinch vs. touch: $F_{(1,5)}=0.3742, P=0.5675$, heat $v s$. low heat: $F_{(1,5)}=1.0350, P=$ 0.3557 ; A7 group: pinch vs. touch: $F_{(1,5)}=0.3585, P=0.5755$, heat vs. low heat: $F_{(1,5)}=0.0225, P=0.8867$; B2 group: pinch vs. touch: $F_{(1,5)}=0.0014, P=0.9719$, heat $v s$. low heat: $F_{(1,5)}=$ 0.3598, $P=0.5747)$.

The fluctuation in fluorescence intensity during the baseline period was as follows: G-CaMP6: A5 group (0.67 $\pm 0.14 \%)$, A7 group $(0.68 \pm 0.15 \%)$, and B2 group (0.62 $\pm 0.14 \%)$; mCherry: A5 group $(0.74 \pm 0.11 \%)$, A7 group $(0.53 \pm 0.12 \%)$, and B2 group $(0.51 \pm 0.11 \%)$. The G-CaMP6 fluorescence intensity in all the groups was rapidly increased in response to an acute nociceptive stimuli but not to a non-noxious control test $(\Delta \mathrm{F} / \mathrm{F}$ values: A5 group: pinch/touch $=7.98 \pm 1.07 / 0.91 \pm 0.12, P$ $<0.001$, heat/low heat $=7.22 \pm 1.41 / 0.98 \pm 0.22, P<0.001$; A7 group: pinch/touch $=8.20 \pm 2.16 / 0.68 \pm 0.22, P=0.0018$, heat/low heat $=6.63 \pm 1.84 / 0.71 \pm 0.19, P=0.014 ;$ B2 group: pinch/touch $=7.97 \pm 0.89 / 0.70 \pm 0.19, P<0.001$, heat/low heat $=9.00 \pm 1.56 / 0.74 \pm 0.27, P<0.001, n=6$, by Sidak's test; Figure 5A). The mCherry fluorescence intensity in all the groups did not significantly change in response to stimuli $(\Delta F / F$ values: A5 group: pinch/gentle $=0.59 \pm 0.13 / 0.70 \pm 0.11$ heat/low heat $=0.70 \pm 0.13 / 0.89 \pm 0.10 ;$ A7 group: pinch/ gentle $=0.79 \pm 0.26 / 0.61 \pm 0.19$, heat/low heat $=0.52 \pm$ $0.11 / 0.51 \pm 0.16$; B2 group: pinch/gentle $=0.62 \pm 0.13 / 0.61 \pm$ 0.16 , heat/low heat $=0.76 \pm 0.12 / 0.63 \pm 0.18, n=6$, by Sidak's test; Figure 5A). The response patterns to a tail pinch and heat stimuli were not significantly different between the three groups; this was shown by the statistical results of onset and peak latencies ( $P>0.05$, by Sidak's test; Figure 5B and $\mathbf{C})$. 


\section{Research Article}
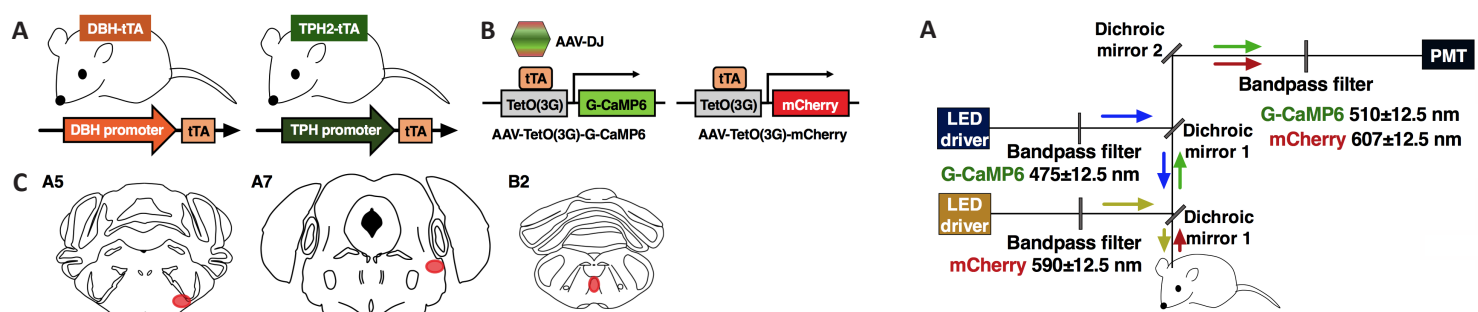

G-CaMP6



C A5
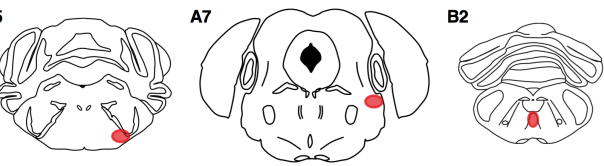

Figure 1 | NA or 5-HT neuron-specific expression patterns of G-CaMP6 or mCherry fluorescent proteins adopting the Tet system.

(A) Genetic scheme of DBH-tTA or TPH-tTA mice, carrying tTA under the control of DBH-tTA or TPH2 promoter, respectively. (B) G-CaMP6 and mCherry fluorescent proteins are expressed using the tTA/TetO system. (C) AAV injection into the target sites (A5, A7, and B2). 5-HT: Serotonin; AAV: adeno-associated virus; $\mathrm{DBH}$ : dopamine $\beta$-hydroxylase; NA: noradrenaline; Tet: tetracycline; TPH: tryptophan hydroxylase; tTA: tetracycline-controlled transactivator transgene.
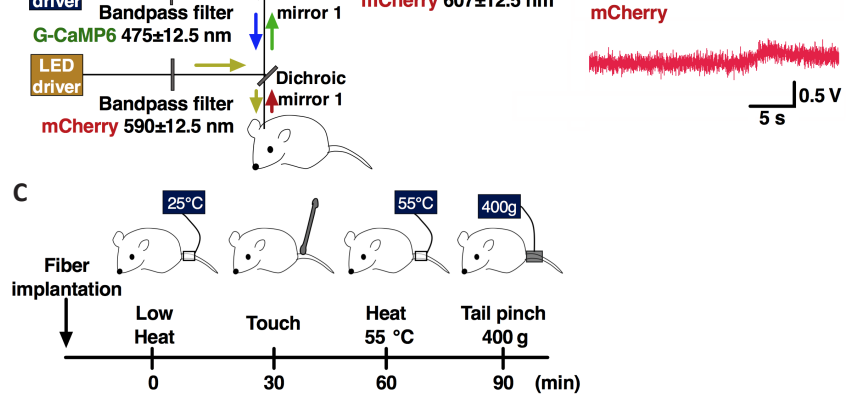

Figure 2 | Experimental procedures.

(A) Schematic representation of the fiber photometry system with two channels. (B) Monitoring of the fluorescence signal intensity. It is confirmed that the fluorescence intensity increases rapidly when the optical position approaches the target sites. (C) Recording procedure. Each of the four stimuli are delivered once to each mouse. We set the inter-stimulus interval to 30 minutes to reduce any possible effects of previous stimuli.

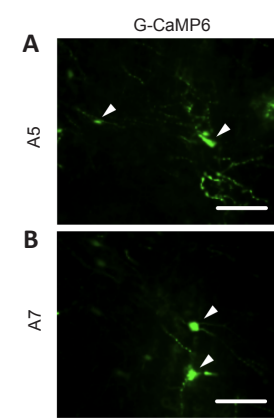

G-CaMP6



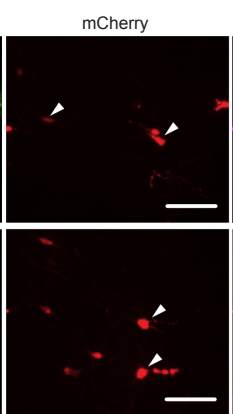

mCherry

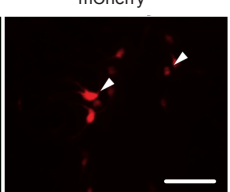

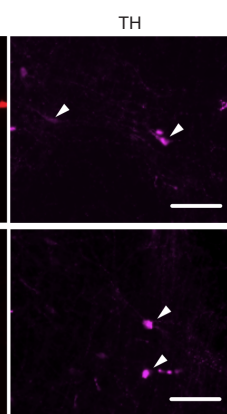

$\mathrm{TPH}$

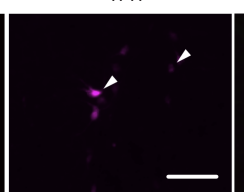

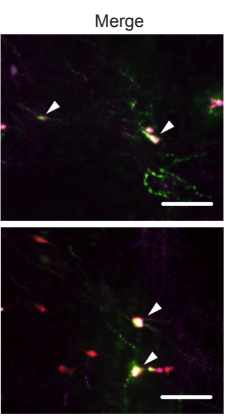

Merge

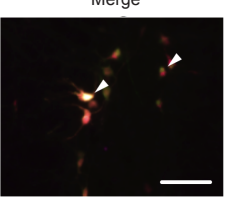

D A5

$\mathrm{G}-\mathrm{T}+$
$9.4 \%$

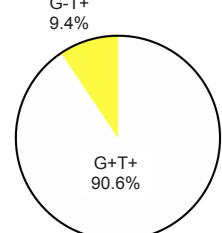

G+T-

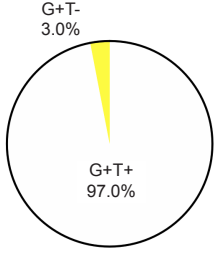

E A7

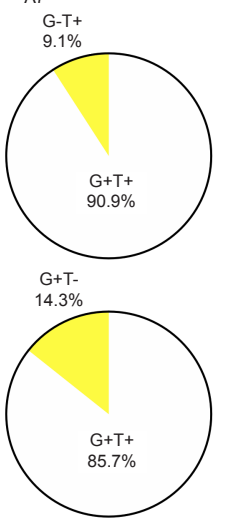

B2

G-T+

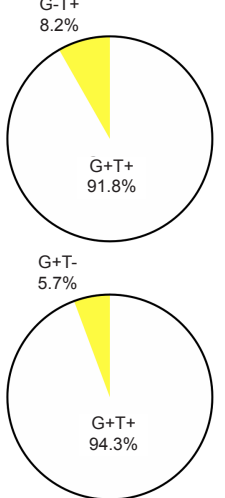

Figure 3 | Expression of AAV-induced G-CaMP6/mCherry.

(A-C) Specific expression patterns of G-CaMP6 (green)/mCherry (red) confirmed in A5/A7 NA neuronal soma (white arrowheads) (A, B) and B2 5-HT neuronal soma (white arrowheads) (C). TH-positive cells (purple) found in the A5 and A7 areas (A, B). TPH-positive cells (purple) found in the B2 area (C). Scale bars: $100 \mu \mathrm{m}$. (D, E) A large proportion of TH-positive cells expressed G-CaMP6 (A5 area: 90.6\%, A7 area: 90.9\%; $n=6$, each). All G-CaMP6-positive cells expressed mCherry and a large proportion of them expressed TH (A5 area: $97.0 \%$, A7 area: $85.7 \%$ ). (F) A large proportion of TPH-positive cells expressed G-CaMP6 (91.8\%; $n=6)$. All G-CaMP6 positive cells expressed mCherry and 94.3\% expressed TPH. 5-HT: Serotonin; AAV: adeno-associated virus; NA: noradrenaline; TH: tyrosine hydroxylase; TPH: tryptophan hydroxylase.

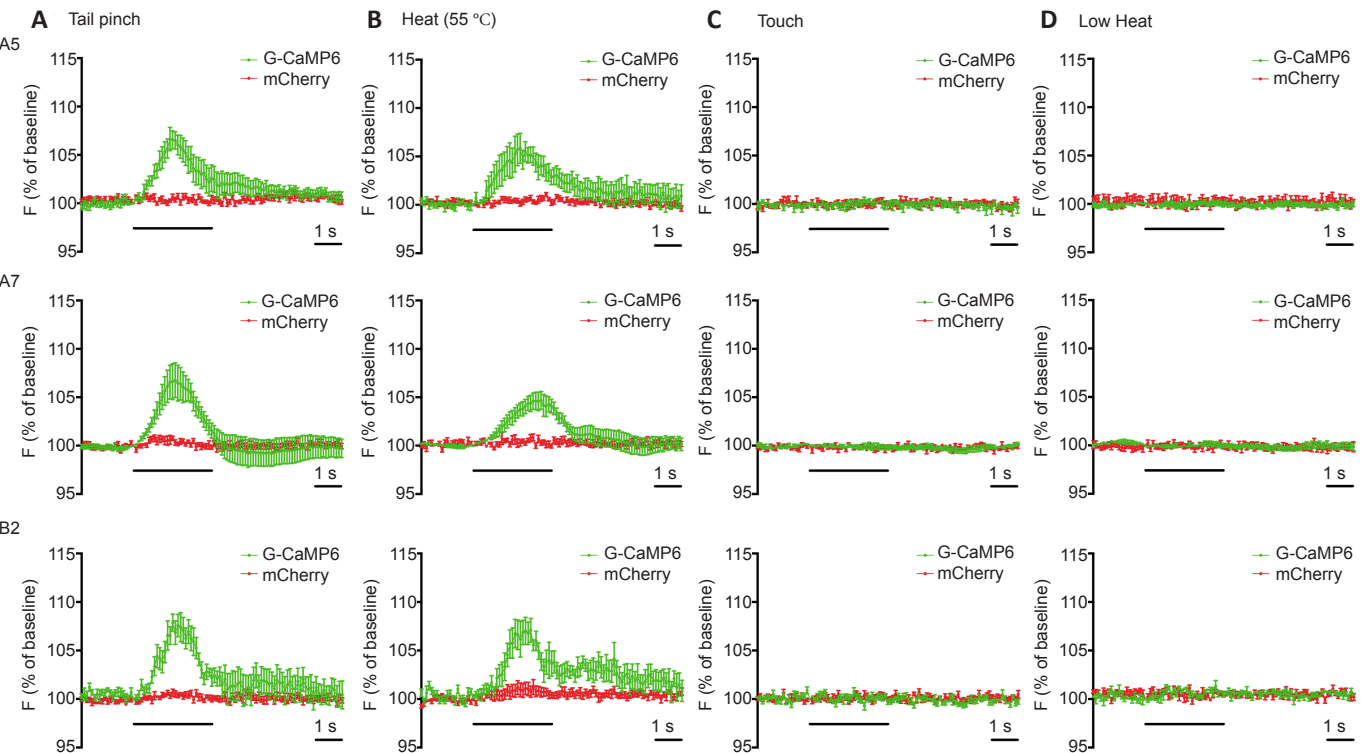

Figure 4 Averaged traces of G-CaMP6/mCherry (green/ red) fluorescence intensity in response to stimuli.

(A, B) Acute nociceptive stimuli or (C, D) a non-noxious control $(n=$ 6, each). G-CaMP6 fluorescence intensity in A5/A7 groups and B2 group is rapidly increased in response to acute nociceptive stimuli but not to non-noxious control stimuli. Short scale bar: 1 second; long scale bar: 3 seconds. 


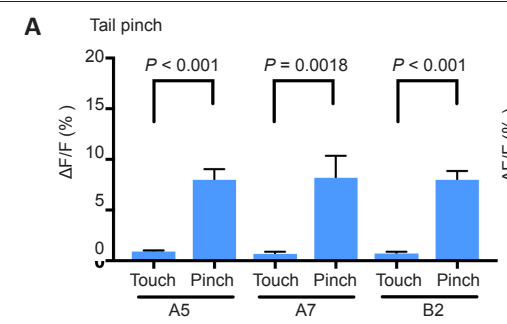

B

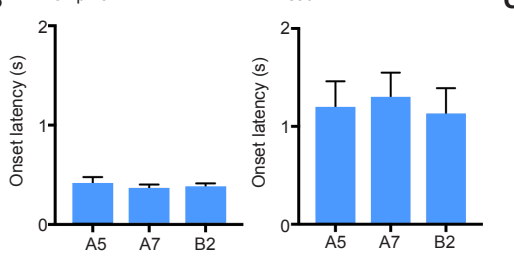

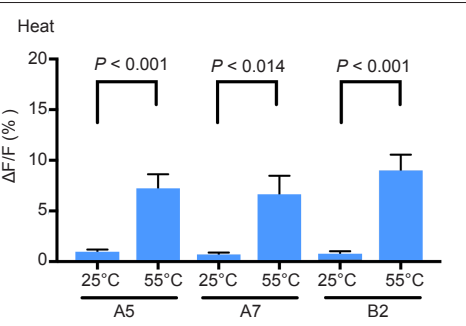

C

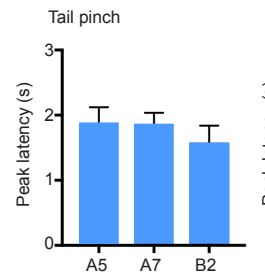

Heat

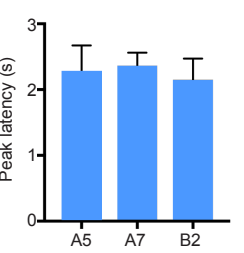

Figure 5 | Effects of acute nociceptive stimuli or a non-noxious control on G-CaMP6/mCherry fluorescence signals.

(A) G-CaMP6 fluorescence intensity in all groups is rapidly increased in response to acute nociceptive stimuli but not to a non-noxious control. (B) The response patterns to a tail pinch and heat stimuli are not significantly different between different groups, as indicated by the statistical results of the onset and peak latency values. Data are expressed as values of the mean \pm SEM and analyzed using a two-way analysis of variance with the Sidak's test ( $n=6$, each).

\section{Discussion}

Our findings clearly demonstrated that the activities of A5/ A7 NA or B2 5-HT neurons were rapidly increased by acute nociceptive stimuli but not by a non-noxious control test. As mentioned earlier, A5 NA neurons are located in the ventrolateral pons and A7 NA neurons are located in the lateral part of the pons, with A5/A7 NA neurons projecting into the spinal cord and forming a part of the pontospinal NAergic system (Proudfit and Clark, 1991). The neuronal population of the NAergic DAS comprised of about $80 \%$ A6, $12 \%$ A5, and $8 \%$ A7 neurons (Howorth et al., 2009). A few studies have demonstrated that A5 NA neurons appeared to be chemically induced during pain (Tavares et al., 1997; Marques-Lopes et al., 2010). Martins et al. (2015) reported that pCREB expression was significantly increased in the A5 site of the spinal nerve injury in animals. Another study reported that the A5-spinal pathway increased noxious-evoked c-Fos expression (Tavares and Lima, 2002). These findings are consistent with our data.

A few studies have also reported that the chemical (Yeomans et al., 1992; Jeong et al., 2012; Wei and Pertovaara, 2013) or electrical (Yeomans and Proudfit, 1992) stimulation of A7 NA neurons regulates nociceptive processing. Some studies have assessed the physiological state of A7 NA cells. To the best of our knowledge, our study is the first study to assess the activities of A5/A7 NA neuronal soma in response to nociceptive stimuli using an electrophysiological method (a fiber photometry system). Our results clearly show a momentary reaction of A5/A7 and lay forth a new perspective concerning DAS.

Regarding the onset latency/peak latency, A5/A7 NA neurons did not show any significant differences, meaning that the response patterns of A5/A7 NA neurons did not depend on the type of stimuli. In a previous study, it was shown that acute nociceptive stimuli rapidly increased the activity of A6 NA neurons in awake mice in real-time conditions measured using a fiber photometry system (Moriya et al., 2019). In this study, the onset latency/peak latency values in the A6 site were as follows: pinch group, $0.4 \pm 0.2 / 1.9 \pm 0.3$, heat group, $0.7 \pm$ $0.1 / 3.5 \pm 0.7$. Our data suggest that A5/A6/A7 NA neurons nearly simultaneously react to acute nociceptive stimuli. In the future, studies comparing these characteristics in the same experimental system will be of interest. In addition, the LC-spinal NAergic system (A6 related) and pontospinal system (A5/A7 related) may work nearly simultaneously in response to acute nociceptive processing. Wei and Pertovaara (2013) reported that the A7 NA neurons affected the A6 NA neurons by projecting their neuronal axons to the $A 6$ area and regulating pain sensitivity. Taking these findings into consideration, our data suggest that the A7-spinal NAergic pathway, A6-spinal NAergic pathway, and A7-A6 NAergic pathway might influence each other. Studies investigating these possibilities will be needed in the future.

The role of B2 5-HT neurons in nociceptive processing is poorly understood. As mentioned in the Introduction, B2 5-HT neurons hardly project into the spinal dorsal horn, mainly targeting the IML nucleus (Allen and Cechetto, 1994; Benarroch, 2014) or the spinal ventral horn (Saruhashi et al., 1996), but regulate the autonomic (Allen and Cechetto 1994; Benarroch, 2014) or motor function (Benarroch, 2014). To the best of our knowledge, our study is the first to assess the activities of B2 5-HT neurons using an electrophysiological method. Our data clearly showed that B2 5-HT neurons were implicated in nociceptive processing. The IML and spinal ventral horn, like B2, are not well understood in the context of nociceptive processing. In addition to DAS, the B2-IML/spinal 5-HTergic pathway may be implicated in nociceptive processing. Studies assessing this will be of interest in the future.

In a previous study, it was confirmed that the activities of B3 5-HT neurons were rapidly increased in response to acute nociceptive stimuli (Moriya et al., 2019). Some electrophysiological studies have mentioned the activation of B3 5-HT neurons in response to nociceptive stimuli (Gautier et al, 2017). As with A5/A7, B2 did not show significant differences in onset latency/peak latency, meaning that the response patterns of B2 5-HT neurons did not depend on the type of stimuli. In our previous paper, onset latency/ peak latency values in B3 were as follows: pinch group, 0.5 $\pm 0.1 / 2.4 \pm 0.5$; heat group, $1.8 \pm 1.0 / 5.2 \pm 1.8$ ) (Moriya et al., 2019). It may be because the B2/B3 5-HT neurons nearly simultaneously react to acute nociceptive stimuli. Future studies assessing this issue will be of interest. Our present results provide new evidence on descending 5-HTergic nociceptive processing.

In this study, we used a fiber photometry system using G-CaMP6/mCherry proteins as intracellular calcium ion indicators. This calcium imaging system enables a high temporal resolution and is not affected by time lag and metabolism; thus, it differs from other electrophysiological and chemical methods. Therefore, our results are robust in that they are the product of momentary and non-medicated data. We chose acute pain over slow pain because we considered it important to appraise momentary fluctuation while using a fiber photometry system. In this study, we did not study responses to slow pain using the formalin or carrageenan test, or the complete Freund's adjuvant model. However, in the future, studies that compare the responses to acute and slow pain are of interest.

Especially in the case of mental disorders, the pathogenetic mechanisms underlying the onset of pain are sometimes unclear. In psychiatry, pain symptoms present in the form of 


\section{Research Article}

neuropathic pain (Attal and Bouhassira, 2019), somatoform pain disorder (Maggio et al, 2020), major depressive disorder (Gebhardt et al., 2016), and sleep-related disorder (Morin et al., 2015). The monoaminergic system in the CNS is thought to be involved in the etiology of these diseases (Nardone et al., 2015; Llorca et al., 2016). In psychiatric clinical medicine, antidepressants are frequently prescribed for pain symptoms, with serotonin noradrenaline reuptake inhibitors (SNRIS) used most frequently. SNRIs mainly act on the synapses of NA neurons in the CNS and take at least a few weeks to improve symptoms (Mitsi and Zaxhariou, 2016). Some studies have suggested that SNRIs inhibit the transmission of nociceptive information (Attal, 2012; Lelic et al, 2016). In theory, a few weeks after starting to take SNRIs, the amount of NA in A5A7 synapses is increased. This may affect the activities of A5/ A7 NA neurons. Besides SNRIs, selective serotonin reuptake inhibitors (SSRIs) or tricyclic antidepressants are also frequently prescribed. SSRIs mainly act on the synapses of 5-HT neurons in the CNS and affect the amount of 5-HT, which may in turn affect the activities of B1/B2 5-HT neurons. We suggest that A5/A7/B2 neurons may represent new therapeutic targets.

There are some limitations to this study. We did not use SNRI/SSRI drugs and therefore did not assess how these drugs affected the activities of A5/A7 NA neurons or B2 5-HT neurons. We focused on pontine nuclei originating in the brain stem but not on the target areas to which they project. In the future, the simultaneous measurement of neuronal activities in nuclear origins and spinal cord will be of interest. In addition, we used acute nociceptive stimuli rather than slow nociceptive stimuli. In the future, studies using chronic stimuli will be needed.

In conclusion, acute nociceptive stimuli rapidly increased the activities of A5/A7 NA or B2 5-HT neurons. This suggests that A5/A7 NA or B2 5-HT neurons play important roles in nociceptive processing in the CNS.

Acknowledgments: We thank all staff members of the Institute of Laboratory Animal Sciences at Kagoshima University for keeping the animals in good condition. We acknowledge the Joint Research Laboratory, Kagoshima University Graduate School of Medical and Dental Sciences, for the use of their facilities.

Author contributions: SM and TK designed the study. SM, AYamashita, JS, YI, $Y S$, and YK conducted the study with the support of AYamanaka and TK. SM and DM analyzed the data and created the figures. SM wrote the manuscript. All authors approved the final version of manuscript.

Conflicts of interest: The authors declare that they have no competing interests.

Financial support: This work was supported by JSPS KAKENHI grants (19K17093 to SM; 20 K06858 to AYamashita; $16 \mathrm{HO5130}$ to TK) and CREST JST (JPMJCR1656 to AYamanaka). The authors have no conflicts of interest to declare.

Institutional review board statement: All performed procedures were approved by the Institutional Animal Use Committee of Kagoshima University (MD17105) on February 22, 2018.

Author statement: This paper has been posted as a preprint on Research Square with DOI:10.21203/rs.3.rs-31869/v1 before submitting, which is available from: $h$ ttps://www.researchsquare.com/article/rs-31869/v1. Copyright license agreement: The Copyright License Agreement has been signed by all authors before publication.

Data sharing statement: Datasets analyzed during the current study are available from the corresponding author on reasonable request.

Plagiarism check: Checked twice by iThenticate.

Peer review: Externally peer reviewed.

Open access statement: This is an open access journal, and articles are distributed under the terms of the Creative Commons AttributionNonCommercial-ShareAlike 4.0 License, which allows others to remix, tweak, and build upon the work non-commercially, as long as appropriate credit is given and the new creations are licensed under the identical terms. Additional files:

Additional file 1: The specific sequences of AAV vectors. Additional Figure 1: Confirmation of fiber implantation.

\section{References}

Allen GV, Cechetto DF (1994) Serotoninergic and nonserotoninergic neurons in the medullary raphe system have axon collateral projections to autonomic and somatic cell groups in the medulla and spinal cord. J Comp Neurol 350:357-366.
Attal N, Bouhassira D (2019) Translational neuropathic pain research. Pain 160:S23-28. Attal N (2012) Neuropathic pain: mechanisms, therapeutic approach, and interpretation of clinical trials. Continuum (Minneap Minn) 18:161-175.

Benarroch EE (2014) Medullary serotonergic system: organization, effects, and clinical correlations. Neurology 83:1104-1111.

Costa AR, Carvalho P, Flik G, Wilson SP, Reguenga C, Martins I, Tavares I (2019) Neuropathic pain induced alterations in the opioidergic modulation of a descending pain facilitatory area of the brain. Front Cell Neurosci 13:287.

Dahlstrom A, Fuxe K (1964) Localization of monoamines in the lower brain stem. Experientia 20:398-399

De Caro C, Cristiano C, Avagliano C, Cuozzo M, La Rana G, Aviello G, Russo R (2020). Analgesic and anti-inflammatory effects of perampanel in acute and chronic pain models in mice: interaction with the cannabinergic system. Front Pharmacol 11:620221.

Gautier A, El Ouaraki H, Bazin N, Salam S, Vodjdani G, Bourgoin S, Hamon M (2017) Lentivira vector-driven inhibition of 5-HT synthesis in B3 bulbo-spinal serotonergic projections Consequences on nociception, inflammatory and neuropathic pain in rats. Exp Neurol 288:11-24.

Gebhardt S, Heinzel-Gutenbrunner M, König U (2016) Pain relief in depressive disorders: a meta-analysis of the effects of antidepressants. J Clin Psychopharmacol 36:658-668.

Hirschberg S, Li Y, Randall A, Kremer EJ, Pickering AE (2017) Functional dichotomy in spinal-vs prefrontal-projecting locus coeruleus modules splits descending noradrenergic analgesia from ascending aversion and anxiety in rats. Elife 6:e29808.

Howorth PW, Teschemacher, AG, Pickering AE (2009) Retrograde adenoviral vector targeting of nociresponsive pontospinal noradrenergic neurons in the rat in vivo. J Comp Neurol $1512 \cdot 141-157$

Inutsuka A, Yamashita A, Chowdhury S, Nakai J, Ohkura M, Tagushi T, Yamanaka A, Kuwaki T (2016) The integrative role of orexin/hypocretin neurons in nociceptive perception and analgesic regulation. Sci Rep 6:29480

Jacobs BL, Martin-Cora FJ, Fornal CA (2002) Activity of medullary serotonergic neurons in freely moving animals. Brain Res Rev 40:45-52.

Jacobs BL, Azmitia EC (1992) Structure and function of the brain serotonin system. Physiol Rev 72:165-229.

Jeong Y, Moes JR, Wagner M, Holden JE (2012) The posterior hypothalamus exerts opposing effects on nociception via the A7 catecholamine cell group in rats. Neuroscience 227:144153

Lelic D, Fischer IW, Olesen AE, Mørch CD, Arguissain FG, Manresa JA, Drewes AM (2016) Venlafaxine and oxycodone effects on human spinal and supraspinal pain processing: randomized cross-over trial. Eur J Neurosci 44:2966-2974

Llorca-Torralba M, Borges G, Neto F, Mico JA, Berrocoso E (2016) Noradrenergic locus coeruleus pathways in pain modulation. Neuroscience 338:93-113.

Maggio J, Alluri PR, Paredes-Echeverri S, Larson AG, Sojka P, Price BH, Perez DL (2020) Briquet syndrome revisited: implications for functional neurological disorder. Brain Commun 2:fcaa156.

Makris UE, Abrams RC, Gurland B, Reid MC (2014) Management of persistent pain in the older patient: a clinical review. JAMA 312:825-836

Marques-Lopes J, Pinho D, Albino-Teixeira A, Tavares I (2010) The hyperalgesic effects induced by the injection of angiotensin II into the caudal ventrolateral medulla are mediated by the pontine A5 noradrenergic cell group. Brain Res 1325:41-52.

Martins I, Carvalho P, de Vries MG, Teixeira-Pinto A, Wilson SP, Westerink BH, Tavares I (2015) Increased noradrenergic neurotransmission to a pain facilitatory area of the brain is implicated in facilitation of chronic pain. Anesthesiology 123:642-653

Melzack R, Wall PD (1965) Pain mechanisms: a new theory. Science 150:971-979.

Mitsi V, Zaxhariou V (2016) Modulation of pain, nociception, and analgesia by the brain reward center. Neuroscience 338:81-92.

Morin CM, Drake CL, Harvey AG, Krystal AD, Manber R, Riemann D, Spiegelhalder K (2015) Insomnia disorder. Nat Rev Dis Primers 1:15026.

Moriya S, Yamashita A, Nishi R, Ikoma Y, Yamanaka A, Kuwaki T (2019) Acute nociceptive stimuli rapidly induce the activity of serotonin and noradrenalin neurons in the brain stem of awake mice. IBRO Rep 7:1-9.

Moriya S, Yamashita A, Masukawa D, Kambe Y, Sakaguchi J, Setoyama H, Yamanaka A, Kuwaki T (2020a) Involvement of supralemniscal nucleus (B9) 5-HT neuronal system in nociceptive processing: a fiber photometry study. Mol Brain 13:14

Moriya S, Yamashita A, Masukawa D, Setoyama H, Hwang Y, Yamanaka A, Kuwaki T (2020b) Involvement of A13 dopaminergic neurons located in the zona incerta in nociceptive processing: a fiber photometry study. Mol Brain 13:60

Mravec B, Bodnar I, Uhereczky G, Kvetnansky R, Palkovits M (2012) Effect of lesions of A5 or A7 noradrenergic cell group or surgical transection of brainstem catecholamine pathways on plasma catecholamine levels in rats injected subcutaneously by formalin. Ge Physiol Biophy 31:247-254

Nardone R, Höller Y, Thomschewski A, Höller P, Lochner P, Golaszewski S, Brigo F, Trinka E (2015) Serotonergic transmission after spinal cord injury. J Neural Transm (Vienna) 122:279-295.

Nuseir K, Proudfit HK (2000) Bidirectional modulation of nociception by GABA neurons in the dorsolateral pontine tegmentum that tonically inhibit spinally projecting noradrenergic A7 neurons. Neuroscience 96:773-783.

Paxionos G, Flanklin K (2019) Paxinos and Flanklin's the Mouse Brain in the Stereotaxic Coordinates, $5^{\text {th }}$ ed. New York: Acadimic Press.

Pertovaara A (2006) Noradrenergic pain modulation. Prog Neurobiol 80:53-83.

Proudfit HK, Clark FM (1991) The projections of locus coeruleus neurons to the spinal cord. Prog Brain Res 88:123-141

Saruhashi Y, Young W, Perkins R (1996) The recovery of 5-HT immunoreactivity in lumbosacral spinal cord and locomotor function after thoracic hemisection. Exp Neurol 139:203-213.

Tavares I, Lima D (2003) The caudal ventrolateral medulla as an important inhibitory modulator of pain transmission in the spinal cord. J Pain 3:337-346.

Tavares I, Lima D, Coimbra A (1997) The pontine A5 noradrenergic cells which project to the spinal cord dorsal horn are reciprocally connected with the caudal ventrolateral medulla in the rat. Eur J Neurosci 9:2452-2461.

Wei H, Pertovaara A (2013) Regulation of neuropathic hypersensitivity by $\alpha(2)$-adrenoceptors in the pontine A7 cell group. Basic Clin Pharmacol Toxicol 112:90-95.

Wessendorf MW, Anderson EG (1983) Single unit studies of identified bulbospinal serotonergic units. Brain Res 279:93-103.

Yaksh TL, Hammond DL, Tyce GM (1981) Functional aspects of bulbospinal monoaminergic projections in modulating processing of somatosensory information. Fed Proc 40:2786-2794. Yeomans DC, Clark FM, Paice JA, Proudfit HK (1992) Antinociception induced by electrical stimulation of spinally projecting noradrenergic neurons in the A7 catecholamine cell group of the rat. Pain 48:449-461.

Yeomans DC, Proudfit HK (1992) Antinociception induced by microinjection of substance P into the A7 catecholamine cell group in the rat. Neuroscience 49:681-691.

C-Editors: Zhao M, Liu WJ, Li CH; T-Editor: Jia Y 

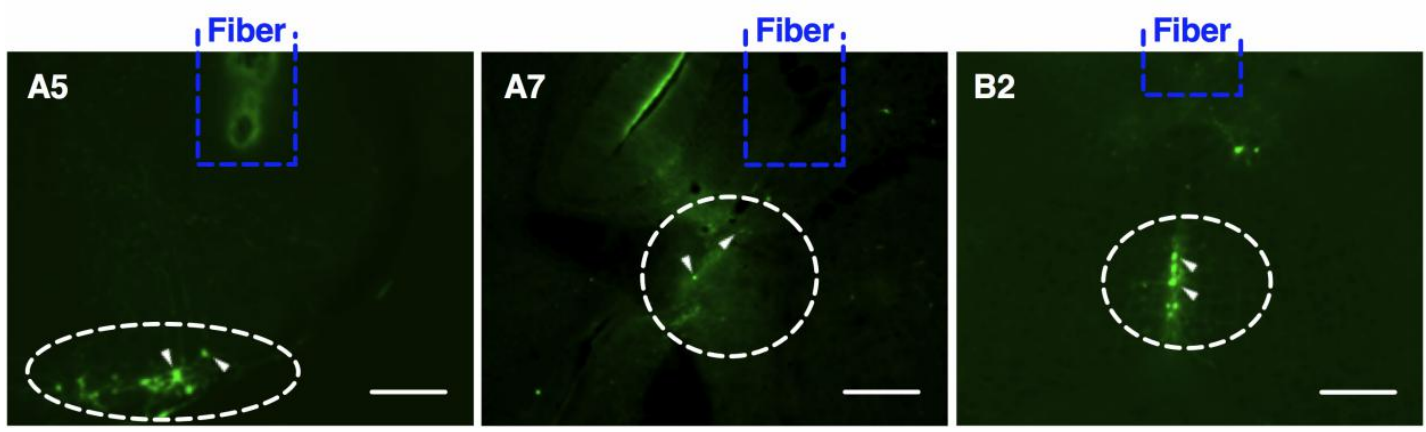

Additional Figure 1 Confirmation of fiber implantation.

The silica fiber (dotted rectangles in blue) located just above the A5, A7, and B2 areas (dotted ovals) that express G-CaMP6 (arrowheads). Scale bars: $300 \mu \mathrm{m}$. 